УДК 621.78.066;669-154;536-33

DOI: $10.17308 / \mathrm{kcmf} .2019 .21 / 714$

Поступила в редакцию 25.11 .2018

Подписана в печать 15.02.2019

\title{
ВЛИЯНИЕ ТЕМПЕРАТУРЫ НА СКОРОСТЬ РАССЛОЕНИЯ РАСПЛАВОВ Sn-Pb В КАПИЛЛЯРАХ
}

\author{
(C) 2019 Н. С. Бусов, Н. П. Углев* \\ ООО КЦН «Матрица» \\ ул. Куйбышева, 47, 614016 Пермь, Россия
}

\begin{abstract}
Аннотация. Проведено исследование влияния температуры на скорость процесса расслоения металлического расплава олово-свинец в наклонном стеклянном капилляре. Основной целью исследования является установление механизма расслоения металлов. Температура считается одним из основных параметров молекулярно-кинетического движения атомов конденсированной системы частиц в жидком состоянии. Скорость движения атомов или кластеров должна определять скорость самого процесса расслоения, если он связан с диффузионными или конвекционными явлениями в системе. Эксперименты показали, что с высокой степенью вероятности температура не влияет на динамику переходных кривых, что указывает на особый механизм этого эффекта, не связанный напрямую с общепринятыми молекулярно-кинетическими закономерностями
\end{abstract}

Ключевые слова: расслоение металлов, кластеры, расслоение в капиллярах, скорость расслоения, барометрическое распределение, молекулярно-кинетический механизм.

\section{ВВЕДЕНИЕ}

Проблема неравномерности состава металлических отливок по высоте, проявляющаяся при выдержке жидких металлических сплавов в литейных формах и тиглях как при производстве отливок, так и при хранении жидкого металла в производственных условиях, имеет не только теоретическое, но и прикладное значение. В работах И. В. Гаврилина $[1,2]$ и наших более поздних исследованиях [3, 4] было установлено влияние ряда факторов на конечные результаты и скорость расслоения компонентов бинарных металлических расплавов при выдержке их в вертикальных или наклонных кварцевых, алундовых или стеклянных капиллярах. Установлено, что распределение компонентов по высоте капилляра асимптотически приобретает вид барометрической кривой, описывающей распределение крупных кластеров (блоков), составленных из разнородных атомов и для вертикальных капилляров практически достигает равновесия за достаточно короткое время порядка 1-2 часов. Для описания этой зависимости может быть использовано барометрическое уравнение Л. Д. Ландау, предложенное для расчёта равновесного распределения в гравитационном поле частиц (молекул) двух сор-

$\bowtie$ Углев Николай Павлович, e-mail: Ouglev@mail.ru тов, имеющих различную плотность $[5,6]$. Увеличение длительности эксперимента практически не изменяет полученный перепад концентрации по концам капилляра $(\Delta C)$, что подтверждает достижение термодинамического равновесия в системе. Установлено, что на скорость расслоения компонентов металлических расплавов оказывают влияние самые разнообразные факторы, связанные с механизмом процесса: длина и диаметр капилляра, форма, положение и угол наклона, характер взаимодействия расплава со стенками капилляра, состав смеси, способ её предварительного перемешивания, наличие механических барьеров для движения частиц внутри капилляра $[4,7]$. Известно, также, что наличие градиента температуры по длине капилляра не сказывается на итоговых результатах расслоения [8].

По рис. 1 можно проследить влияние длины вертикального капилляра на скорость расслоения $[1,9]$. При длине капилляра $220 \mathrm{~mm}$ предельный перепад достигается за $150 \mathrm{~min}$, поэтому изучение динамики расслоения возможно только в этом периоде. При этом необходимо иметь в виду, что объективным значением скорости расслоения может быть только отношение текущей степени расслоения $\left(\Delta C_{\tau}\right)$ к предельной асимптотической величи- 
не $\Delta C$, зависящей от ряда параметров. В частности, по рис. 1 можно сделать вывод, что скорость расслоения при коротком капилляре $(h=50 \mathrm{~mm})$ значительно выше, чем для длинного $(220 \mathrm{~mm})$, поскольку практически заканчивается на 90 минуте. Таким образом, длительность достижения предельного уровня расслоения, при сохранении всех остальных параметров эксперимента неизменными, может служить характеристикой скорости расслоения.

Наиболее важную роль в любой конденсированной системе частиц имеет температура. Её уровень определяет подвижность отдельных атомов, молекул и кластеров, и, соответственно, динамику перехода системы частиц из одного равновесного состояния в другое, поскольку при увеличении температуры увеличивается коэффициент диффузии и уменьшается вязкость - такие изменения этих параметров приводят к увеличению как микрофизической, так и макрофизической (конвекционной) относительной подвижности компонентов. Одновременно при увеличении температуры снижается глубина барометрического расслоения, что выражается уменьшением $\Delta C$. Однако следует иметь в виду, что уменьшение степени расслоения в этом случае не имеет отношение к скорости процесса, a, как указано выше, связано с термодинамическим равновесием. В то же время, влияние температуры на скорость расслоения ранее не изучалось, хотя эта информация имеет определяющий характер, и напрямую связана с механизмом процесса. В самом деле, - если скорость расслоения увеличивается с повышением температуры, то механизм процесса связан с молекулярно-кинетическими закономер- ностями движения атомов, в том числе и с вязкостью; в противном случае - следует искать альтернативные механизмы.

\section{ОПИСАНИЕ ЭКСПЕРИМЕНТА}

Основной задачей эксперимента является сравнение переходных кривых расслоения сплава, находящегося в одинаковых условиях, но при разных температурах.

Процесс расслоения в вертикальных капиллярах протекает достаточно быстро. В работах И. В. Гаврилина показано, что его результаты заметны уже через 5 минут после начала процесса [1], при том, что при введении капилляра в печь граница жидкости продвигается по высоте капилляра сверху вниз за 1-2 минуты, что определяет, в основном, и полное время прогрева образца до заданной температуры. Это создаёт определённые методические затруднения при исследовании динамики расслоения, внося элемент неопределённости в начало процесса, особенно при его малой длительности. Наклон капилляра ускоряет процесс расслоения в 2-3 раза [4]. Ориентируясь на эти данные, нами были выбраны стандартные длительности процесса 10, 20, 40 и 80 минут, однако принципиальное значение для достижения цели данного исследования имеют результаты «расслоения» только в первые 10-15 минут. В качестве модельного объекта исследования выбран расплав $\mathrm{Sn}-\mathrm{Pb}$, как наиболее исследованный в отношении процесса расслоения.

Исследование динамики расслоения проводили при пяти температурах (таблица), длина и диаметр образцов сплава во всех капиллярах состав-

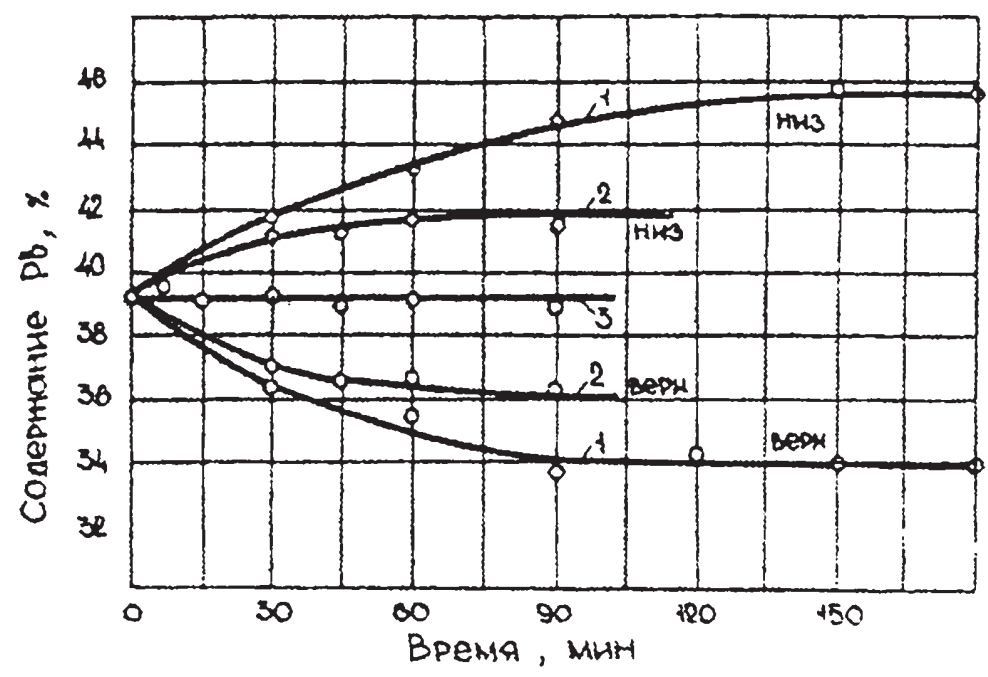

Рис. 1. Расслоение сплава $\mathrm{Pb}-\mathrm{Sn}$ в вертикальном капилляре: $1-h=220 \mathrm{~mm} ; 2-h=50 \mathrm{Mm}$; 3 - горизонтальный капилляр $[1,9]$

[Fig. 1. Pb-Sn alloy stratification in vertical capillary: $1-h=220 \mathrm{~mm} ; 2-h=50 \mathrm{~mm} ; 3$ - horizontal capillary [1,9]] 
ляла 100 и $1.95 \mathrm{~mm}$ соответственно. Для снижения погрешности при каждой температуре и времени эксперимент проводили в 3 или 2 капиллярах одновременно. В таблицу экспериментальных результатов включены начальные условия - столбец при длительности процесса равной нулю (отсутствие перепада концентрации) для всех исследованных температур.

\section{МЕТОДИКА ИССЛЕДОВАНИЯ}

В наклонную печь электросопротивления, разогретую до заданной температуры, помещали пучок одинаковых стеклянных капилляров, заполненных сплавом $\mathrm{Sn}-\mathrm{Pb}(40 \%$ wt.) и герметизированных с обоих концов. Печь и, соответственно, капилляры находились под наклоном $45^{\circ}$ к горизонту. По

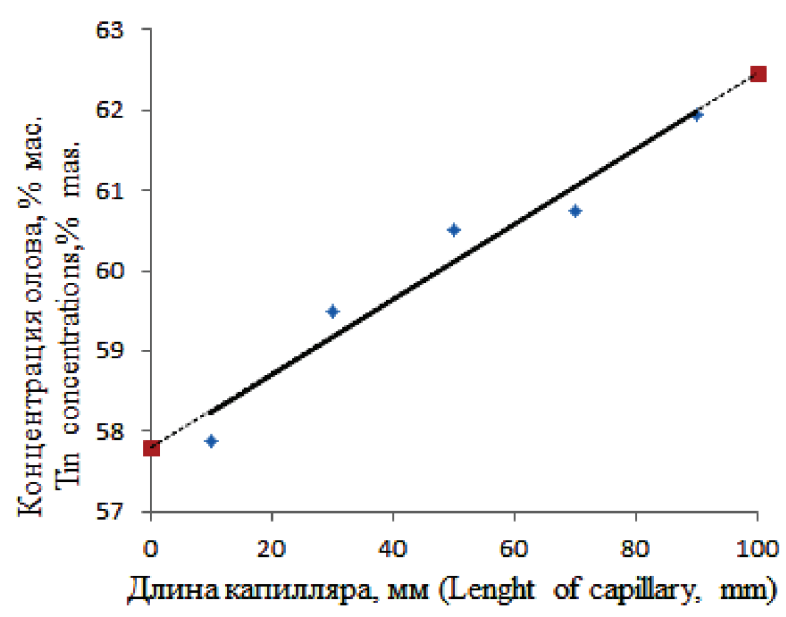

Рис. 2. Распределение концентрации олова по высоте капилляра (среднее по трём измерениям) при температуре $450{ }^{\circ} \mathrm{C}$ и длительности 20 мин, и аппроксимация на всю длину капилляра

[Fig. 2. The distribution of the concentration of tin at the height of the capillary (average of three measurements) at a temperature of $450^{\circ} \mathrm{C}$ and duration of $20 \mathrm{~min}$, and the approximation on the entire length of the capillary] истечении каждого заданного времени извлекали из печи по 3 капилляра, переводили их в горизонтальное положение до полного застывания расплавленного металла. После высвобождения твердых образцов из стеклянной оболочки разрезали их по высоте на 5 одинаковых фрагментов и определяли состав последних рентгенофлюоресцентным методом по заранее построенной калибровочной шкале. Методика эксперимента и анализа достаточно подробно представлены в работе [8]. Погрешность определения по свинцу составляла не более $0.11 \%$ wt. По данным трёх измерений определяли средние значения, на основании которых проводили аппроксимацию результатов расслоения уравнением прямой линии (рис. 2). Таким образом, весь массив экспериментальных данных по составам составлял около 300 измерений. Достигнутый перепад концентрации на полной длине образцов определяли вычислением по аппроксимирующим уравнениям прямой линии. Полные результаты эксперимента представлены в таблице.

По данным таблицы уже можно сделать вывод о том, что основной интенсивный период расслоения во всех случаях проходит в интервале от 0 до 10 минут.

Результаты измерений, представленные в таблице, за исключением двух выпадающих результатов (зачеркнуты), аппроксимировали с помощью программы Table Curve 3D двухпараметрическим уравнением:

$$
\begin{gathered}
\Delta C=7.232+4.188 \ln (t)-0.086 T- \\
-0.773(\ln (t))^{2}+3.176 \cdot 10^{-4} T^{2}+0.002 \cdot T \cdot \ln (t)- \\
-0.001 \cdot(\ln (t))^{3}-4.01 \cdot 10^{-7} \cdot T^{3}-1.113 \cdot 10^{-7} \cdot T^{2} \cdot \ln (t)+ \\
+2.902 \cdot 10^{-6} T(\ln (t))^{2},
\end{gathered}
$$

где $t$ - длительность выдержки, min, $T$ - температура, ${ }^{\circ} \mathrm{C}$, позволяющем построить сглаженные переходные кривые процесса сегрегации от

Таблица. Усреднённые результаты расслоения в зависимости от длительности и температуры процесса

[Table. Averaged results of a stratification depending on the duration and temperature of the process]

\begin{tabular}{|c|c|c|c|c|c|}
\hline $\begin{array}{c}\text { Tемпература, } \\
\begin{array}{c}{ }^{\circ} \mathrm{C} \\
\text { [Temperature, } \\
\left.{ }^{\circ} \mathrm{C}\right]\end{array}\end{array}$ & $\begin{array}{r}\text { Достигнутый перепад концентрации свинца по концам капилляра (\% мас.) } \\
\text { при длительности процесса расслоения, мин } \\
\text { [The achieved difference in the concentration of lead at the ends of the capillary (\% } \\
\text { wt.) for the duration of the lamination process, min] }\end{array}$ \\
\cline { 2 - 6 } & 0 & 10 & 20 & 40 & 80 \\
\hline 210 & 0 & 5.279 & 2.325 & 5.459 & 6.141 \\
\hline 250 & 0 & 6.387 & 6.512 & 3.614 & 4.184 \\
\hline 310 & 0 & 7.464 & 6.988 & 5.890 & 4.591 \\
\hline 350 & 0 & 4.903 & 7.101 & 7.053 & 5.184 \\
\hline 450 & 0 & 3.809 & 4.686 & 3.824 & 4.520 \\
\hline
\end{tabular}


времени при различных температурах с использованием всей полученной базы данных по «расслоению». На рис. 3 в качестве примера построены расчётные кривые динамики расслоения для двух крайних температур эксперимента.

Полученные расчётные переходные кривые имеют качественно одинаковый вид с данными $[1,9]$, представленными на рис. 1.

\section{ОБСУЖДЕНИЕ РЕЗУЛЬТАТОВ ЭКСПЕРИМЕНТА}

Основным выявленным результатом является факт слабого, или полного отсутствия влияния температуры (в пределах погрешности эксперимента) на скорость установления равновесного распределения компонентов в капилляре. Уместно отметить, что состояние равновесия в системе во всех случаях устанавливается за время, меньше 25 минут.

Первоначальной достаточно долго обсуждавшейся и окончательно принятой причиной расслоения металлических расплавов было предположение об осаждении кластеров тяжёлого компонента под воздействием центробежных [10], или гравитационных $[1,11,12]$ сил. Однако эта гипотеза содержала ряд серьёзных противоречий (например, по скорости осаждения кластера в среде, состоящей из плотной смеси кластеров), что позволило поставить её под сомнение и предложить иной механизм [3], заключающийся в предположении об адсорбции одного из компонентов на внутренней поверхности капилляра с последующим течением этого слоя вверх или вниз из-за отличия его плотности от средней плотности расплава. Позднее возникли сомнения и в этом механизме [13], что привело к отказу от него в пользу другого, основанного на гипотезе двумерного мономолекулярного газа из атомов металлов, сосредоточенного в межфазном пространстве между расплавом и стенкой капилляра, и обладающего достаточно экзотичным свойством сверхтекучести $[4,14]$.

Отвлекаясь пока от последнего гипотетического механизма, рассмотрим более понятный вариант «расслоения», основанный на течении адсорбированного слоя.

Расслоение в этом случае протекает в три этапа: сначала атомы или кластеры, например, тяжелого металла, являющегося ПАВ, должны переместиться за счёт диффузии или конвекции из объёма образца к внутренней стенке капилляра; затем по стенке проходит достаточно быстрое течение плёнки вниз; после чего тяжелый компонент внизу

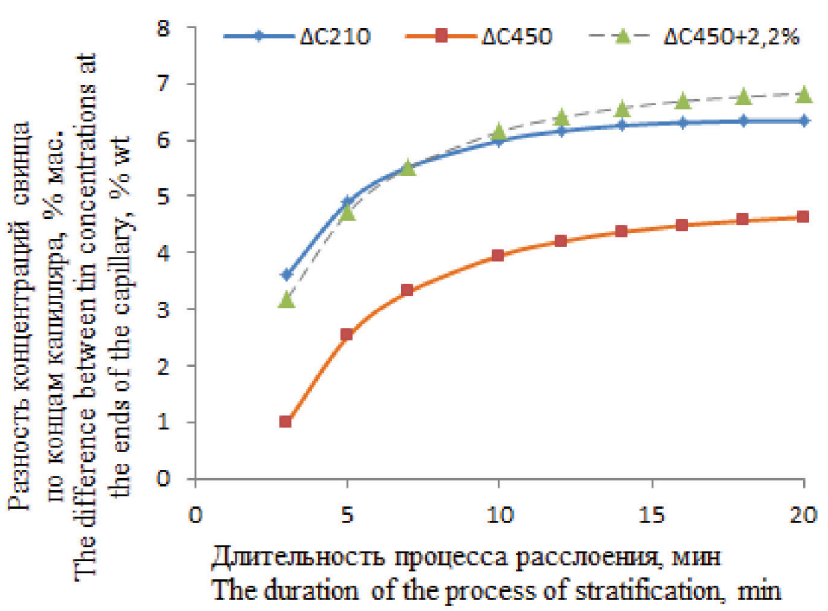

Рис. 3. Изменение перепада концентрации олова по концам капилляров во времени при выдержке их при различной температуре в наклонной печи $(\Delta C 210-$ при $210{ }^{\circ} \mathrm{C} ; \Delta C 450$ - при $450{ }^{\circ} \mathrm{C}$; Наложение (пунктир) $-\Delta C 450+2.2 \%)$

[Fig. 3. Changing the difference in the concentration of tin at the ends of the capillaries over time at their expo-

sure at different temperatures in an inclined furnace $\left(\triangle C 210-\right.$ at $210{ }^{\circ} \mathrm{C} ; \Delta C 450-$ at $450{ }^{\circ} \mathrm{C}$; Overlay (dotted line) $-\Delta C 450+2.2 \%)]$

должен переместиться в объём образца. Поскольку второй компонент не может обладать такими же поверхностно-активными свойствами, его концентрирование в верхней части капилляра может проходить только балансным способом - весь ушедший с тяжелым компонентом объём будет замещаться за счёт медленного вытеснения расплава снизу. Очевидно, что при таком механизме скорость процесса, исчисляемая как скорость изменения концентрации компонентов, должна быть одинакова как для верха, так и для низа капилляра. Иначе говоря, активные фазы процесса могут закончиться только за равные периоды времени, одновременно, а кривые, отражающие динамику расслоения (рис. 1), должны быть объёмно-симметричными. Фактически, в эксперименте И. В. Гаврилина (рис. 1), для капилляра высотой 220 mm получен иной результат - в верхней части капилляра (нижняя кривая) процесс расслоения закончился на 90-й минуте, в то время как внизу (верхняя кривая) - только на 150-й минуте. Это указывает на независимое движение атомов свинца и олова в этом процессе.

Поскольку путь поперёк капилляра значительно меньше, чем вдоль него, диффузия или конвекция могут справиться с требуемой величиной потока, чтобы обеспечить большой расход в приповерхностном слое, однако, при увеличении диаметра капилляра это условие должно всё более за- 
трудняться, поскольку увеличивается средний путь диффузии по радиусу. Следовательно, при увеличении диаметра капилляра следует ожидать снижения скорости расслоения. В действительности, результат противоположный [4] - выявлено, что при увеличении диаметра капилляра скорость расслоения увеличивается практически пропорционально диаметру.

При перераспределении металлов по высоте капилляра за счёт «расслоения» вдоль него возникают продольные градиенты концентрации компонентов, которые вызывают обратные диффузионные потоки по всему сечению образца. Это должно приводить к некоторому снижению достигнутого перепада концентраций. Прямое сравнение скоростей расслоения и диффузии показывает, что при низких температурах диффузионный поток более чем на порядок уступает потоку атомов в процессе расслоения [7]. Учитывая большую разницу скоростей, можно ожидать, что при низких температурах быстрый процесс «расслоения» может привести к «проскоку» динамического равновесия, которое потом устанавливается через какое-то время за счёт развития процесса диффузии, направленной в обратную сторону. При этом разнонаправленное влияние температуры указывает на принципиально различающиеся механизмы и «движущие силы» этих конкурирующих процессов.

Известно, что при изменении температуры от 299 до $622{ }^{\circ} \mathrm{C}$ коэффициент самодиффузии олова увеличивается в 3.6 раза [15]. Динамическая вязкость олова в диапазоне $240-500{ }^{\circ} \mathrm{C}$ падает в 1.6 раза, а свинца в диапазоне $340-550{ }^{\circ} \mathrm{C}$ - в $1.3-$ 1.4 раза [16]. Примерно так же (в 1.47 раз) уменьшается и вязкость сплава $\mathrm{Pb}-\mathrm{Sn}$ (50 \% at.) [16].

Если механизм процесса расслоения металлов в капиллярах определяется процессами диффузии и конвекционного массопереноса, то при увеличении температуры от 210 до $450{ }^{\circ} \mathrm{C}$ (таблица) следует ожидать его ускорения, как минимум, в 1.53.6 раза, что должно проявиться на форме переходных кривых (рис. 3). В частности, переходная кривая, как это и указано выше, при низких температурах должна быть более пологой, а при высокой температуре - более крутой, чего не наблюдается в эксперименте - переходные кривые в начальном периоде процесса от 0 до 20 минут при всех исследованных температурах практически одинаковы, что указывает на несущественное влияние её на этот процесс.

Таким образом, учитывая вышеперечисленные особенности процесса расслоения, можно сделать независимое заключение о том, что механизм сегрегации металлов в капиллярах не связан с молекулярно-кинетическими процессами движения отдельных атомов или кластеров расплава, а является особым полумикроскопическим механическим процессом, определяемым поведением крупных структур жидкости в гравитационном или центробежном поле.

\section{ВЫВОДЫ}

1. Процесс «расслоения» металлических расплавов $\mathrm{Sn}-\mathrm{Pb}$ в стеклянных капиллярах является чрезвычайно быстрым и приходит в состояние равновесия не более чем за 20 минут для наклонных капилляров внутренним диаметром 2 mm при длине $100 \mathrm{~mm}$.

2. Температура не оказывает влияния на скорость достижения равновесия в системе

3. Эксперимент подтверждает, что механизм расслоения бинарного металлического расплава $\mathrm{Sn}-\mathrm{Pb}$, содержащего наноразмерные кластеры разных составов, не связан с общепринятыми молекулярно-кинетическими закономерностями движения отдельных атомов или более крупных объектов.

\section{ИСТОЧНИК ФИНАНСИРОВАНИЯ}

Исследование выполнено при финансовой поддержке РФФИ, проект № 16-01-00662a.

\section{КОНФЛИКТ ИНТЕРЕСОВ}

Авторы декларируют отсутствие явных и потенциальных конфликтов интересов, связанных с публикацией настоящей статьи.

\section{СПИСОК ЛИТЕРАТУРЫ}

1. Гаврилин И. В. // Изв. АН СССР. Металль, 1984, № 3, с. 191-193.

2. Гаврилин И. В. // Изв. АН СССР. Металль, 1985, № 2, с. 66-73.

3. Углев Н. П. // Изв. АН СССР. Металльь, 1987, № 2, c. $45-46$.

4. Углев Н. П., Углев С. Н. // Конденсированные среды и межфазные гранищы, 2014, т. 16, № 4, с. 508512. URL: http://www.kcmf.vsu.ru/resources/t_16_4_2014_ 014.pdf

5. Ландау Л. Д., Лифшиц Е. М. Теоретическая физика: Учебное пособие. В 10 m. T.V. Статистическая физика. М.: Наука, 1964, 568 с.

6. Углев Н. П. «Синтез знаний в естественных науках. Рудник будущегго: проекть, технологии, оборудование», материалы Междунар. науч. конф., 2011, Пермь, 2011, т. 2, с. 557-563.

7. Углев Н. П., Углев С. Н. // Расплавы, 2018, № 4, c. 411-419. DOI: 10.1134/S023501061804014X 
8. Углев Н. П., Дирякова Е. Ю. // Металльы, 2015, № 4, c. 26-29.

9. Гаврилин И. В. Плавление и кристаллизация металлов и сплавов. Владимирский гос. ун-т, Владимир, $2000,260 \mathrm{c}$.

10. Бунин К. П. // Изв. АН СССР, ОТН, Металлургия и топливо, 1946, № 2, с. 305-307.

11. Дутчак Я. И., Клым Н. М. // Физика Металлов и Металловедение, 1965, т. 19, № 1, с. 137.
12. Новохатский И. А., Архаров В. И. // Докл. АН СССР, Химия, 1971, т. 201, № 4, с. 905-908. B86.

13. Углев Н. П. Деп. ВИНИТИ, 30.12.86, № 9026-

14. Углев Н. П. // Расплавы, 2017, № 1, с. 72-82.

15. Вилсон Д. Р. Структура жидких металлов и сплавов. М.: Металлургия, 1972, 247 с.

16. Андронов В. Н, Чекин Б. В, Нестеренко С. В. Жидкие металлы и илаки. Справочник. М.: Металлургия, 1977, $128 \mathrm{c}$.

\title{
THE INFLUENCE OF TEMPERATURE ON THE SEPARATION RATE OF SN-PB MELTS IN CAPILLARIES
}

\author{
(C) 2019 N. S. Boosov, N. P. Ouglev* \\ LLC KCN "Matrix" \\ 47 Kuibyshev srt. 614016 Perm, Russian Federation
}

\begin{abstract}
Objective. When holding binary metal melts in vertical capillaries, the effect of their partial functional stratification can be observed.The equilibrium height distribution of a heavy component corresponds to the barometric dependence and is achieved in less than 2 hours. A further increase in the duration of the stratification process does not lead to an increase in the degree of achieved segregation. It is shown that the obtained distribution can be well described by the Landau parametric equation for a mixture of two types of particles with different densities. The particle size determined by the approximation of experimental data for $\mathrm{Sn}-\mathrm{Pb}$ melts by the Landau equation corresponds to clusters containing 8.000-10.000 atoms.

The speed of the stratification process depends on many factors: the height and the diameter of the capillary, the inclination of the capillary, the composition of the mixture, the interaction of the melt with the material of the capillary, and other parameters. It is known that the temperature is the main determining factor of the molecular kinetic motion of particles in a liquid. For example, an increase in temperature leads to an increase in diffusion and a decrease in viscosity. Therefore, if the process of stratification is associated with the molecular-kinetic movement, the increase in temperature should result in the acceleration of the stratification process. The aim of the experiment was to study the dependence of the current concentration difference at the ends of the capillary on the duration of stratification (stratification rate) at different temperatures.

Methods and methodology. The process of stratification of $\mathrm{Sn}-\mathrm{Pb}$ eutectic mixtures was carried out in inclined capillaries of the same length and diameter at different temperatures. The comparison of the obtained transition curves showed that the processes of stratification reach equilibrium in the same time for all given temperatures (from 210 to $450^{\circ} \mathrm{C}$ ). This indicates that the stratification rate is independent of the process temperature.

Conclusion. Therefore, it can be concluded that the mechanism of the stratification process of metal melts in capillaries is not related to the classic molecular kinetic motion of atoms in the system. This result is crucial for the study of properties of the liquid state and requires the development of a special theory to explain it.
\end{abstract}

Keywords: stratification of metals, clusters, stratification in capillaries, stratification rate, barometric distribution, molecular kinetic mechanism.

Ouglev Nikolai P., e-mail: ouglev@mail.ru. 


\section{SOURCE OF FINANCING}

The reported was supported by the Russian Foundation for Basic Research (grant No. 16-01-00662a).

\section{CONFLICT OF INTEREST}

The authors declare the absence of obvious and potential conflicts of interest related to the publication of this article.

\section{REFERENCES}

1. Gavrilin I. V., Frolova T. B., Zaharov V. P. Izv. $A N$ SSSR. Metally, 1984, no. 3, pp. 191-193. (in Russ.)

2. Gavrilin I. V. Izv. AN SSSR. Metally, 1985, no. 2, pp. 66-73. (in Russ.)

3. Uglev N. P. Izv. AN SSSR. Metally, 1987, no. 2, pp. 45-46. (in Russ.)

4. Uglev N. P., Uglev S. N. Condensed Matter and Interphases, 2014, vol. 16, no. 4, pp. 508-512. URL: http://www. kcmf.vsu.ru/resources/t_16_4_2014_014.pdf (in Russ.)

5. Landau L. D., Lifshic E. M. Teoreticheskaya fizika. vol.5. Statisticheskaya fizika [Theoretical Physics. Textbook. In 10 Vol. 5. Statistical Physics]. Moscow, Nauka Publ., 1964, 568 p. (in Russ.)

6. Uglev N. P. "Sintez znanij v estestvennykh naukakh", sbornik materialov Mezhdunarodnoi nauch. Konferencii",
Perm. gos. nats. issl. un-t; Perm, 2011, vol. 2, pp. 557-563. (in Russ.)

7. Uglev N. P., Uglev S. N. Rasplavy, 2018, no. 4, pp. 411-419. DOI: $10.1134 /$ S023501061804014X

8. Uglev N. P., Diryakova E. Yu. Metally, 2015, no. 4, pp. 26-29. (in Russ.)

9. Gavrilin I. V. Plavlenie i kristallizaciya metallov $i$ splavov [Melting and Crystallization of Metals and Alloys]. Vladimirskij gos. un-t., Vladimir., 2000, 260 p. (in Russ.)

10. Bunin K. P. Izv. AN SSSR. OTN. Metallurgiya $i$ toplivo, 1946, no. 2, pp. 305-307. (in Russ.)

11. Dutchak Ya. I., Klym N. M. Fizika Metallov $i$ Metallovedenie, 1965, vol. 19, no. 1, p. 137. (in Russ.)

12. Novokhatskij I. A., Arkharov V. I. Dokl. AN SSSR, Himiya, 1971, vol. 201, no. 4, pp. 905-908. (in Russ.)

13. Uglev N. P. Dep. VINITI, 30.12.86, no. 9026-B86. (in Russ.)

14. Uglev N. P. Rasplavy, 2017, no. 1, pp. 72-82. URL: https://elibrary.ru/item.asp?id=28999157 (in Russ.)

15. Vilson D. R. Struktura zhidkikh metallov $i$ splavov [Structure of Liquid Metals and Alloys]. Moscow, Metallurgiya Publ., 1972, 247 p. (in Russ.)

16. Andronov V. N., Chekin B. V., Nesterenko S. V. Zhidkie metally i shlaki . [Liquid Metals and Slags. Handbook]. Spravochnik, Moscow, Metallurgiya Publ., 1977, 128 p. (in Russ.)
Углев Николай Павлович - к. х. н., доцент кафедры химических технологий, Пермский национальный исследовательский политехнический университет (ПНИПУ), научный руководитель ООО «КЦН «Матрица», Пермь, Российская Федерация; e-mail: ouglev@mail.ru. ORCID iD 00000002-1734-2256.

Бусов Никита Сергеевич - бакалавр химикотехнологического факультета, кафедры химических технологий, Пермский национальный исследовательский политехнический университет (ПНИПУ), Пермь, Российская Федерация; e-mail: azazaryi@yandex.ru. ORCID iD 0000-0001-7560-0842.
Ouglev Nikolai P. - Cand. Sci (Chem.), Associate Professor, Chemical Technology Department, Perm National Research Polytechnic University, Scientific Adviser of "Matrix", Perm, Russian Federation; e-mail: ouglev@mail.ru. ORCID iD 0000-0002-1734-2256.

Boosov Nikita S. - bachelor, Chemical Technology Department, Perm National Research Polytechnic University, Perm, Russian Federation; e-mail: ouglev@ mail.ru. ORCID iD 0000-0001-7560-0842. 